\title{
Parallel Analysis of Fluorescence Detection on Multi- channel Capillary Electrophoresis Microchip
}

\author{
Zhihuan Liu, Weiping Yan, Xiaobo Yang, Hongfeng Lv, Zhenan Tang \\ School of Electronic Science and Technology, Dalian University of Technology, \\ Dalian 116024, P. R. China \\ yanwp@dlut.edu.cn
}

\begin{abstract}
:
A simple and compact parallel analysis of fluorescence detection system with cross-polarization was presented and applied to multi-channel capillary electrophoresis. This device was demonstrated using a array of surface mounted devices light-emitting diode (SMD LED) as the excitation light source and a linear charge coupled device (CCD) as a photodetector. A cost-effective cross-polarization scheme was employed to eliminate almost all of the excitated light. With the combination of polarizer and $C C D$, the provided system could detect different concentrations of one dye or multiple dyes simultaneously because of the capability of $C C D$ and polarizers responding to the wavelength of visible light. This system has been used for fluorescence detection of capillary electrophoresis microchip with Rhodamine 6G, Rhodamine B and fluorescein and the limit-of-detection were $1 \mu \mathrm{M}$, $10 \mu \mathrm{M}, 100 \mu \mathrm{M}$ respectively. The project opens a door to integrate high-sensitivity microchip with light source and detector for inexpensive and relatively simple fluorescence-based analytical system.
\end{abstract}

Key words: parallel analysis, cross-polarization, multi-channel microchip, liner charge coupled device and light-emitting-diode

\begin{abstract}
Introduction
Chemical sensors based on sensitive detection of light intensity have been widely used in medical diagnoses, biological research, environmental monitoring, and drug screening as analytical tools [1]. Although more and more detection schemes including chemilluminescence $(C L)[2,3]$, electrochemistry (EC) [4], electro spray mass spectrometry (ESMS) [5], and nuclear magnetic resonance (NMR) [6] have been developed in the past few years, fluorescence detection is still the most common diagnostic method in micro scale biological and chemical analysis due to high sensitivity, and is becoming increasingly important for the need of low-cost and disposable lab-on-a-chip (LOC) system.
\end{abstract}

A critical challenge in developing an integrated fluorescence detection system in typical detector geometry is how to filter out the excitation light which has overlapped with the fluorescent dyes emission spectrum from the detector. Recently, a variety of techniques have been tried from the standpoints of low-cost and integration, such as interference filters [7-9], color filters [10,11] and dye-based filters [12], but all of these filters are specific to particular dye wavelengths and must be matched to the particular integrated system.

One attraction of microfludic chip is the ability to increase throughput by employing multichannel chip, which is desirable for high-throughput genetic and proteomic analysis as well as drug screening. However, with the higher throughput microfluidic chips, more complicated problems came out for the needs of much more bulky optics and sophisticated electrical connections $[13,14]$. There is a great demand for low-cost, compact integrated multi-channel fluorescence detection system that can process many requests in parallel.

A layer-by-layer multispectral fluorescence detection system was designed by making main components to thin-plane style. The resultant devices would offer a low-cost solution for chemical and biological parallel processing analysis.

\section{Experimental}

Experimental setup

A simple and compact multi-spectral fluorescence detection system was established for parallel analysis with multichannel CE chip. The schematic diagram of the experimental 
setup is demonstrated in Fig. 1. It mainly consisted of green and blue surface mounted devices LED array (the peak emitting wavelength are $532 \mathrm{~nm}$ and $464 \mathrm{~nm}$, respectively) as excitation light source, two sets of polarizes, high-voltage power supply, glass/PDMS multichannel CE microchip, and a CCD sensor as detector. The glass/PDMS multi-channel CE microchip was sandwiched between two crossaligned polarizes. Polarizer 1, located between LED array layer and multi-channel CE chip, changed the light from LED array to be linear polarization. While the other one, polarizer 2, located under the microfluidic chip, oriented at $90^{\circ}$ to polarizer 1 , filtered out the excitation light and only permitted the fluorescence signals to pass through to the CCD sensor. In order to reduce the disturbance of adjacent channels, a piece of black film with three pinholes (diameter of $400 \mu \mathrm{m}$ ) corresponding to the detection area of the microchannels was inserted between the polarizer 2 and CCD detector. A 12 bit, $20 \mathrm{MHz}$ sampling frequency $A / D$ card was connected between CCD sensor and a computer to collect the fluorescence signals, and a program written by Visual $\mathrm{C}++$ 6.0 was used for data acquisition and display.

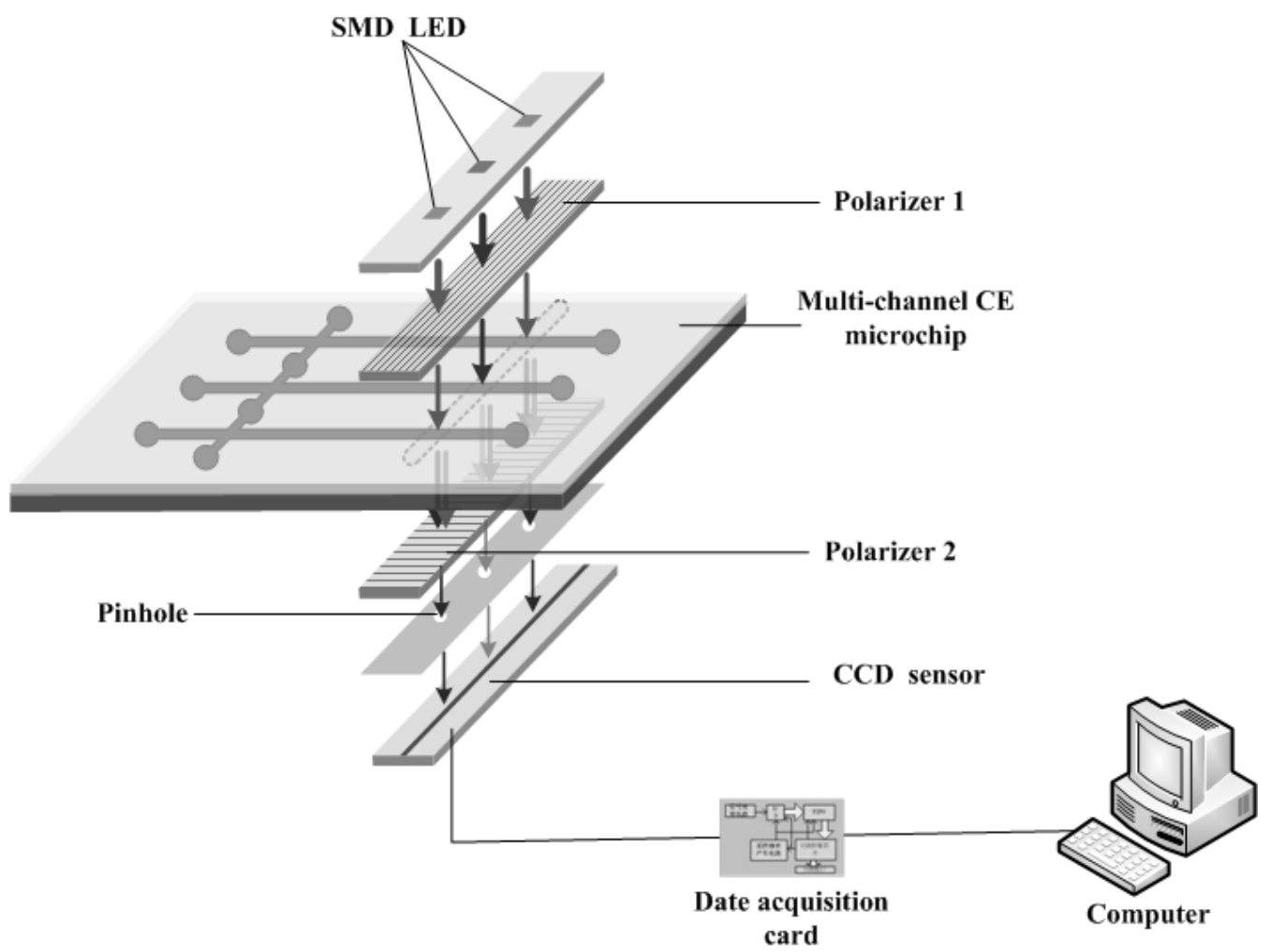

Fig. 1. Schematic illustration of the experimental setup for multispectral fluorescence detection system.

\section{Chemicals}

All chemicals in the following experiments were analytical grade, and deionized (DI) water was used throughout. Fluorescent dyes including fluorescein, Rhodamine $6 \mathrm{G}$ and Rhodamine B were purchased from LDSJ (Dalian, China). PDMS (Sylgard 184) original solution was obtained from Dow Corning Ltd. (USA). Different concentrations of samples were diluted from $5 \mathrm{mM}$ stock solutions of prepared in absolute ethanol. The running buffer solution tris-borate-EDTA (TBE) was prepared by dissolving a solid TBE mixture in DI water to a final concentration of $89 \mathrm{mM}$ tris (hydroxymethyl) aminomethane, $89 \mathrm{mM}$ boric acid and $2 \mathrm{mM}$ ethylenediaminetetraacetic acid (pH 8.3).

\section{Microfludic chip}

The multi-channel $C E$ chips used in the following experiments were designed and fabricated in-house. The glass substrate with microchannel network was manufactured by standard photolithography and wet chemical etching techniques. The cover plate was a piece of $1 \mathrm{~mm}$ thick PDMS replica from a flat glass wafer. PDMS (Sylgard 184) was mixed at a $10: 1(\mathrm{~m} / \mathrm{m})$ ratio of elastomer base to curing agent, poured onto the wafer, degassed to remove air bubbles from the polymer mixture in a vacuum oven and cured at $80{ }^{\circ} \mathrm{C}$ for 150 minutes. Immediately PDMS was sealed to the glass substrate after being peeled off the wafer.

As shown in Fig. 2, the micro CE chip contains ten reservoirs and three set of separation 
channels. The injection and separation channels were $10 \mathrm{~mm}$ and $40 \mathrm{~mm}$ long respectively, and the distance from injection point to the detection point was approximately $30 \mathrm{~mm}$ long. The distance between two separation channels is $12 \mathrm{~mm}$ and the volume of reservoirs is $5 \mu \mathrm{L}$. All the channels designed in the chip are typically $60 \mu \mathrm{m}$ deep and 100 $\mu \mathrm{m}$ wide.

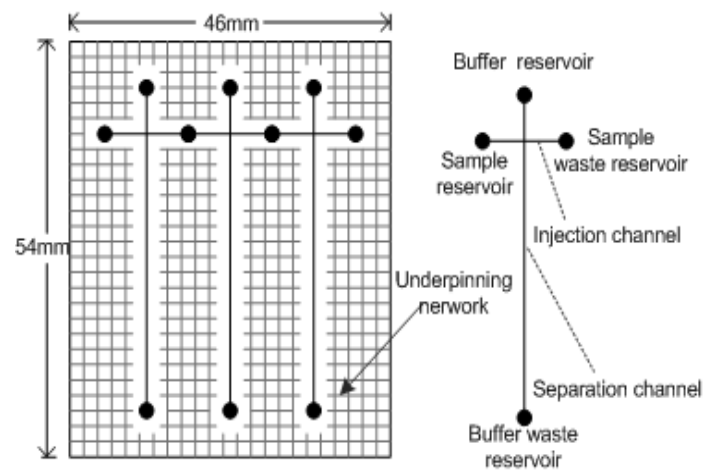

Fig. 2. Layout of the multi-channel CE chip. The detailed view of a single channel is shown on the right.

Injection and separation

The microchannels were activated with $1 \mathrm{M}$ sodium hydroxide, deionized water (18 M $\mathrm{M}$ ) and TBE running buffer prior to use. The microdevice was operated with two modes as shown in Fig. 3, sample injection and separation mode. For the injection step, electrokinetic injection was accomplished by applying potential to the sample reservoirs for a period of time with a home-made high-voltage power supply. During this phase, the analyte is electrokinetically driven from $S$ (grounded) to SW (600v) with B and BW remaining afloat for $15 \mathrm{~s}$. Then the power supply was switched, and the sample was separated during the migration from B (grounded) toward BW (1400V) with S and SW afloat to prevent leakage of analyte to the separation channels. After separation, the microchannels were immediately rinsed with absolute ethanol to prevent them from being blocked.

\section{Results and discussion}

Polarization isolation

To characterize the degree of polarization isolation with different excitation light, blue, green and red LEDs were employed as the original light. The extinction ratio was obtained by testing the intensity of initial light and polarized light after the polarizers.

As shown in Fig.4 less than $0.0003 \%$ original light were transmitted from the crosspolarization approach, which corresponded to a nominal extinction ratio of $\sim 47 \mathrm{~dB}$. Since most of the excitation light eliminated by the polarizers, the $\mathrm{S} / \mathrm{N}$ ratio of the provided system was greatly improved. However, the polarizers do cripple the signal intensity of fluorescent dye.

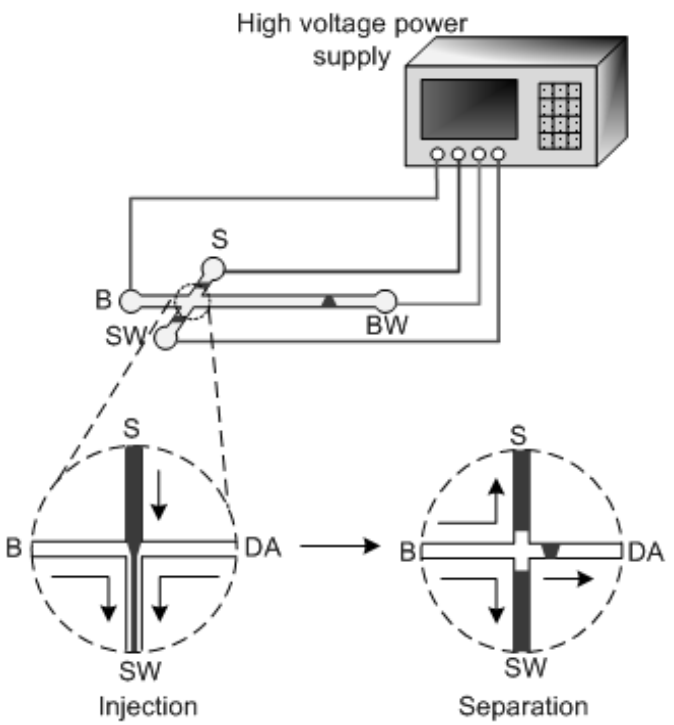

Fig. 3. Schematic illustration of injection and separation modes on a single channel. B indicates buffer reservior, $S$ indicates sample reservoir, $S W$ indicates sample waste reservior, and DA indicates detection area.

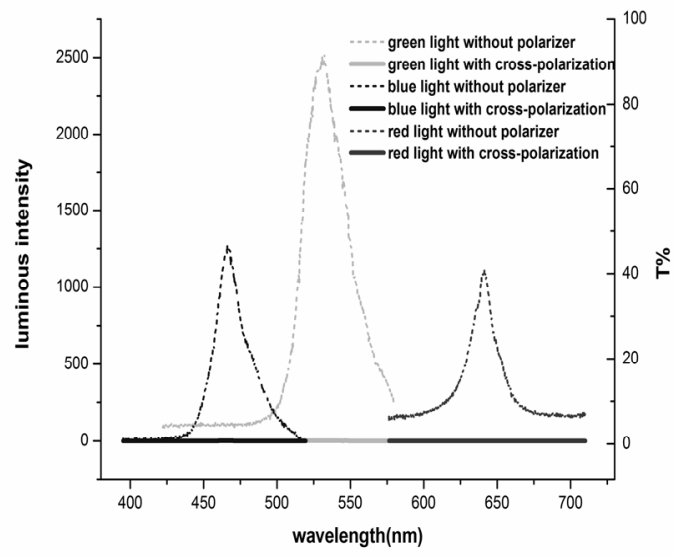

Fig. 4. Optical characteristics of cross-polarization.

Concentration measurements

The multi-spectral fluorescence detection system has been used for fluorescence detection of three-channel CE with fluorescein, Rhodamine $6 G$ and Rhodamine $B$ at the same time. As shown in Fig. 5, with the concentration of $10^{-3} \mathrm{~mol} / \mathrm{L}$, the fluorescent intensity of Rhodamine $6 \mathrm{G}$ was stronger than fluorescein and Rhodamine $\mathrm{B}$ under the same condition. A limit-of-detection of $1 \mu \mathrm{M}, 10 \mu \mathrm{M}$ and $100 \mu \mathrm{M}$ was demonstrated for Rhodamine 6G, Rhodamine B and fluorescein. The provided system could bring different dyes' signals clearly out of the excitation light noise. 


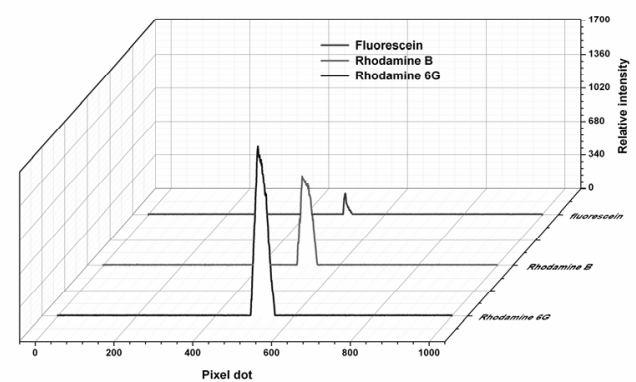

Fig. 5. Fluorescence detection with fluorescein, Rhodamine $6 G$ and Rhodamine $B$ in parallel on three-channel CE microchip.

\section{Conclusions}

In conclusion, a novel, simple and inexpensive multi-spectral fluorescence detection system was established. The background signals were substantially reduced as the cross-polarization scheme eliminated the noise of excitation light successfully, thereby significantly enhancing the sensitivity of the system. This parallel analysis fluorescence detection approach was applied to electrophoresis with fluorescein, Rhodamine $6 \mathrm{G}$ and Rhodamine $\mathrm{B}$. With this device, different dyes can be processed simultaneity. The results proved that multispectral fluorescent detection can be realized with appropriate combination of polarizers and CCD detector. The assembled system offers an attractive route towards the fabrication of integrated, low-cost detector for quantitative relatively simple fluorescence-based analytical system, which is not only suited to commonly chemical analysis, but could also be applied to environmental monitoring and point-of-care medical diagnostics.

\section{Acknowledgements}

The research was supported by National Natural Science Foundation of China (Grant No. 61074166 and 61131004$)$ projects.

\section{References}

[1] R. Narayanaswamy and O. S. Wolfbeis, Optical Sensors for Industrial, Environmental and Clinical Applications (Springer Series on Chemical Sensors and Biosensors), 4 (2004); ISBN-13: 978-3540408864

[2] Shakuntala D. Mangru and Dr. D. Jed Harrison, Chemiluminescence detection in integrated postseparation reactors for microchip-based capillary electrophoresis and affinity electrophoresis, Electrophoresis 19, 2301-2307 (1998); doi: 10.1002/elps. 1150191309

[3] Xuhua Wang, Maliwan Amatatongchai, Duangai Nacapricha et al., Tin-film organic photodiodes for integrated on-chip chemiluminescence detection - application to antioxidant capacity screening, Sensors and Actuators B: Chemical 140, 643-648 (2009); doi:

10.1016/j.snb.2009.04.068

[4] Adam T. Woolley, Kaiqin Lao, Alexander N. Glazer et al, Capillary Electrophoresis Chips with Integrated Electrochemical Detection, Anal. Chem. 70, 684-688 (1998); doi: 10.1021/ac971135z

[5] Jianjun Li, Pierre Thibault, Nicolas H. Bings et al., Integration of Microfabricated Devices to Capillary Electrophoresis-Electrospray Mass Spectrometry Using a Low Dead Volume Connection: Application to Rapid Analyses of Proteolytic Digests, Anal. Chem. 71, 3036-3045 (1999); doi: 10.1021/ac981420y

[6] Henk Wensink, Fernando Benito-Lopez, Dororhee $\mathrm{C}$. Hermes et al, Measuring reaction kinetics in a lab-on-a-chip by microcoil NMR, Lab Chip 5, 280-284 (2005); doi: 10.1039/B414832k

[7] J. R. Webster, M. A. Burns, D. T. Burke, and C. H. Mastrangelo, Monolithic capillary electrophoresis device with integrated fluorescence detector, Anal. Chem. 73, 1622-1626 (2001); doi: 10.1021/ac0004512

[8] M. A. Burns, B. N. Johnson, S. N. Brahmasandra et al., An integrated nanoliter DNA analysis device, Science 282, 484-487 (1998); doi: 10.1126/science.282.5388.484

[9] Bo Yao, Guoan Luo, Liduo Wang et al., A microfluidic device using a green organic light emitting diode as an integrated excitation source, Lab on a Chip 5, 1041-1047 (2005); doi: 10.1039/b504959h

[10] Sean J. Hart, Renee D.Jiji, A simple, low-cost, remote fiber-optic micro volume fluorescence flowcell for capillary flow-injection analysis, Anal. Bioanal.Chem. 374, 385-389 (2002); doi: 10.1007/s00216-002-1525-2

[11] Haifeng Li, Jinming Liu, Rongguo Su et al., A compactly integrated laser-induced fluorescence detector for microchip electrophoresis, Electrophoresis 25, 1907-1915 (2004); doi: 10.1002/elps.200305867

[12] Oliver Hofmann, Xuhua Wang, Alastair Cornwell et al. Monolithically integrated dye-doped PDMS long-pass filters for disposable on-chip fluorescence detection, Lab on a Chip 6, 981-987 (2006); doi: 10.1039/b603678c

[13] Zheng Shen, Xiao Liu, Zhicheng Long et al., Parallel analysis of biomolecules on a microfabricated capillary array chip, Electrophoresis 27, 1084-1092 (2006); doi: 10.1002/elps.200500689

[14] X. Yang, W. Yan, H. Bai et al., A scanning laser induced fluorescence detection system for capillary electrophoresis microchip based on optical fiber, Optik - Int. J. Light Electron Opt. (2011), doi:10.1016/j.jileo.2011.10.016 